\title{
Miniature standoff Raman probe for neurosurgical applications
}

\author{
Oliver A. C. Stevens \\ Joanne Hutchings \\ William Gray \\ Rosa Louise Vincent \\ John C. Day
}




\title{
Miniature standoff Raman probe for neurosurgical applications
}

\author{
Oliver A. C. Stevens, ${ }^{a, \star}$ Joanne Hutchings, ${ }^{a, b}$ William Gray, ${ }^{c}$ Rosa Louise Vincent, ${ }^{d}$ and John C. Day ${ }^{a}$ \\ aUniversity of Bristol, Interface Analysis Centre, H.H. Wills Physics Laboratory, Tyndall Avenue, Bristol BS8 1TL, United Kingdom \\ ${ }^{\mathrm{b} B i o p h o t o n i c s ~ R e s e a r c h ~ U n i t, ~ G l o u c e s t e r s h i r e ~ H o s p i t a l s ~ N H S ~ F o u n d a t i o n ~ T r u s t, ~ G l o u c e s t e r ~ G L 1 ~ 3 N N, ~ U n i t e d ~ K i n g d o m ~}$ \\ ${ }^{\circ}$ Cardiff University, Neuroscience and Mental Health Research Institute, Schools of Medicine and Biosciences, Cardiff CF14 4XN, United Kingdom \\ dPfizer, 230 East Grand Avenue, South San Francisco, California 94080, United States
}

\begin{abstract}
Removal of intrinsic brain tumors is a delicate process, where a high degree of specificity is required to remove all of the tumor tissue without damaging healthy brain. The accuracy of this process can be greatly enhanced by intraoperative guidance. Optical biopsies using Raman spectroscopy are a minimally invasive and lower-cost alternative to current guidance methods. A miniature Raman probe for performing optical biopsies of human brain tissue is presented. The probe allows sampling inside a conventional stereotactic brain biopsy system: a needle of length $200 \mathrm{~mm}$ and inner diameter of $1.8 \mathrm{~mm}$. By employing a miniature standoff Raman design, the probe removes the need for any additional components to be inserted into the brain. Additionally, the probe achieves a very low internal silica background while maintaining good collection of Raman signal. To illustrate this, the probe is compared with a Raman probe that uses a pair of optical fibers for collection. The miniature stand-off Raman probe is shown to collect a comparable number of Raman scattered photons, but the Raman signal to background ratio is improved by a factor of five at Raman shifts below $\sim 500 \mathrm{~cm}^{-1}$. The probe's suitability for use on tissue is demonstrated by discriminating between different types of healthy porcine brain tissue. ๑ The Authors. Published by SPIE under a Creative Commons Attribution 3.0 Unported License. Distribution or reproduction of this work in whole or in part requires full attribution of the original publication, including its DOI. [DOI: 10.1117/1.JBO.21.8.087002]
\end{abstract}

Keywords: Raman; probe; brain; biopsy; background; spectroscopy; tumor.

Paper 160229PR received Apr. 10, 2016; accepted for publication Jul. 11, 2016; published online Aug. 10, 2016.

\section{Introduction}

Removal of intrinsic brain tumors, particularly gliomas, is a challenging process, and requires careful consideration to selectively and completely remove tumor tissue, thus improving outcome without damage to healthy brain. ${ }^{1}$ Surgical guidance is currently assisted by a variety of imaging techniques, such as pre- or intraoperative magnetic resonance imaging (MRI), computerized tomography $(\mathrm{CT})$, intraoperative ultrasound, 5-aminolevulinic acid (5-ALA) fluorescent imaging, ${ }^{2}$ and reflectance spectroscopy, ${ }^{3}$ many of which have been shown to improve patient prognosis ${ }^{4,5}$ by ensuring maximal resection of radiologically identified tumor. Optical biopsies using Raman spectroscopy have been demonstrated to be a less invasive and potentially more effective alternative to traditional biopsy for a number of different applications, ${ }^{6-8}$ and potentially offer a reliable and lower cost method of identifying residual tumor intraoperatively, as well as defining apparent tumor edges. ${ }^{9}$ This is especially relevant to glial tumors in which edges are invasive and indistinct. In addition, an alternative to traditional biopsy is particularly important for pontine tumors due to their location in the brain stem, where any removal of tissue carries a risk of irreversible damage to the patient.

To perform "optical biopsies" in vivo, small Raman spectroscopy probes are typically required to efficiently collect a spectrum in situ. A variety of such probes for different applications have been constructed, and shown to be effective in providing spectra information of clinical value. ${ }^{10-19}$

*Address all correspondence to: Oliver A. C. Stevens, E-mail: o.stevens@ bristol.ac.uk
Optical fibers are an efficient method of guiding light where physical dimensions are constrained; for this reason, the majority of compact Raman probes employ fibers to carry the laser light to the sample and the Raman scattered photons back to the spectrometer. However, these fibers impart a background Raman spectrum of silica onto the spectrum of the sample, and hence Raman background caused by silica is an issue that affects most Raman probes. ${ }^{20}$ This silica background is shown in Fig. 1. The silica background is present at all Raman shifts, but is particularly prevalent at low wavenumbers. While background subtraction can be performed after collection of the spectra, this masks the problem rather than truly removing it, and care must be used in its application to prevent artefacts that confound statistical analysis occurring on the spectra. Additionally, as the total number of background counts is increased for a given amount of Raman signal, the shot noise is also increased, leading to a reduced signal-to-noise ratio (SNR). This problem can be avoided by restricting analysis to higher wavenumber regions (above $800 \mathrm{~cm}^{-1}$ ), ${ }^{21,22}$ away from the silica fluorescence, but this risks losing some valuable chemical information at lower wavenumbers. Brain tissue in particular contains peaks potentially useful for classification at Raman shifts of $400 \mathrm{~cm}^{-1},{ }^{23}$ and possibly lower.

There are a number of techniques that can be used to reduce the background in a probe while still meeting the requirements for small dimensions. Some groups have used hollow-core waveguides to reduce the silica signal, ${ }^{24,25}$ but as these use a metal coating to guide the light rather than total internal reflection with an associated attenuation of the signal, resulting in long (>100 s) acquisition times. 


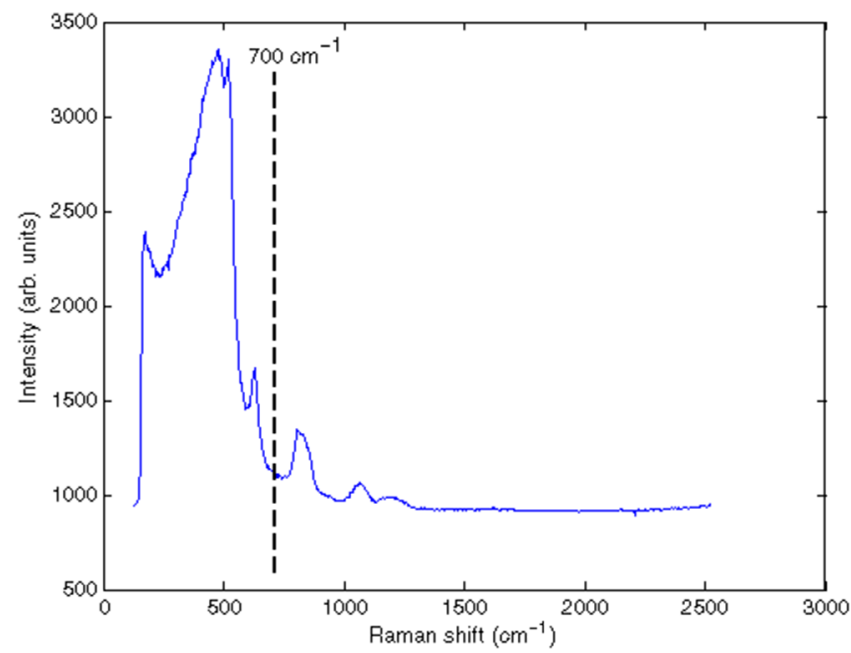

Fig. 1 Silica background present in most Raman probes. Spectrum collected from the tip of an optical fiber imaged on a desktop Raman microsope (Renishaw RM1000, $633 \mathrm{~nm}, \sim 5 \mathrm{~mW}, 1 \mathrm{~s}$ ). The background is present throughout all Raman shifts but is particularly large below $700 \mathrm{~cm}^{-1}$

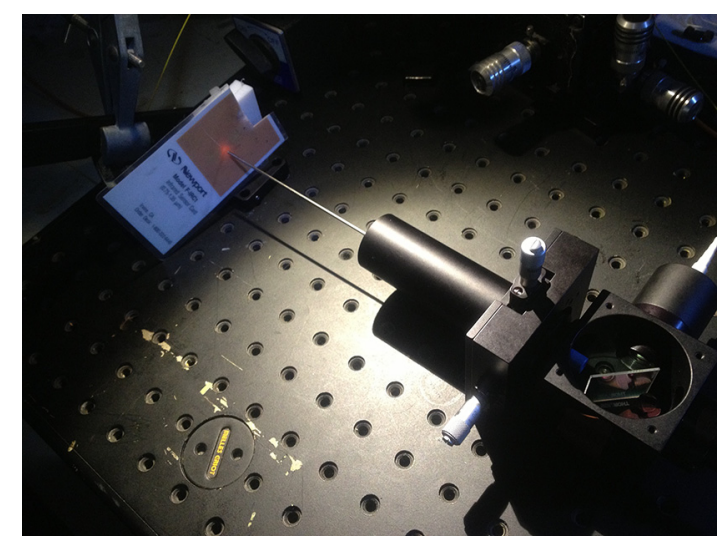

Fig. 2 Operational assembled probe pointed at an IR viewing card.
Hollow-cored photonic crystal fibers (PCF) have been used $^{26,27}$ to replace the excitation and collection fibers, but these are expensive and difficult to work with due to small core diameters and limitations of the range of wavelengths they will accept.

In this paper, we demonstrate a miniature Raman probe design that uses an alternative approach: reducing the background by avoiding the use of fibers in the main section of the probe altogether, thus reducing the internal Raman background but also eliminating the need for additional components to be inserted into the brain.

\section{Design and Construction}

The probe is designed to collect Raman scattered light from within a standard stereotactic brain biopsy needle (AD-TECH medical). A picture of the assembled probe is shown in Fig. 2. The stereotactic needle is $200-\mathrm{mm}$ long and 2-mm diameter.

The optical configuration of the probe is shown in Fig. 3. The probe employs a miniaturized version of "standoff" Raman probe, replacing the optical fibers with a collimated beam to direct the light down the biopsy needle. It uses a single- or few-mode fiber connected to an aspheric lens (Thor Labs model C430TME-B) for collimation of the excitation laser. This serves to reduce divergence within the probe length, and produce the desired $1.8-\mathrm{mm}$ spot at the sample.

Throughput of a probe is defined largely by its optical geometry, or etendue. This is a measure of the total light flux that can pass through an optical system. Etendue is conserved in any optical system, so the element of the design with the smallest etendue limits the overall light throughput; for this probe, this is the hollow needle section, and for many other probes, it is the optical fibers.

The etendue $(G)$ captures this total flux by summing the total area $(A)$ and range of solid angles $(\theta)$ that light can pass through in an optical system.

This is represented below. The elemental etendue $\mathrm{d}^{2} G$ of a system is the product of the elements of area $\mathrm{d} A$ and $\mathrm{d} \Theta$. The total etendue is found by integrating this over all angles and $\operatorname{area}^{28}$

$\mathrm{d}^{2} G=\mathrm{d} A \mathrm{~d} \Theta$

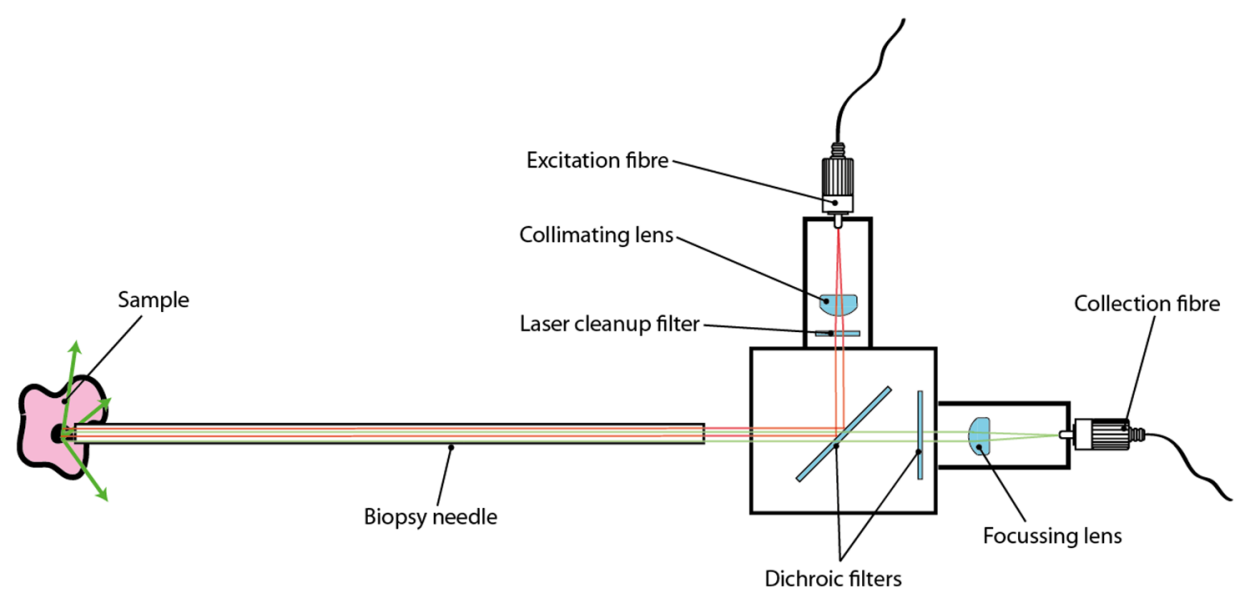

Fig. 3 Optical layout of the probe. The design requires no components in the biopsy needle, greatly increasing medical compatability and reducing Raman background. 


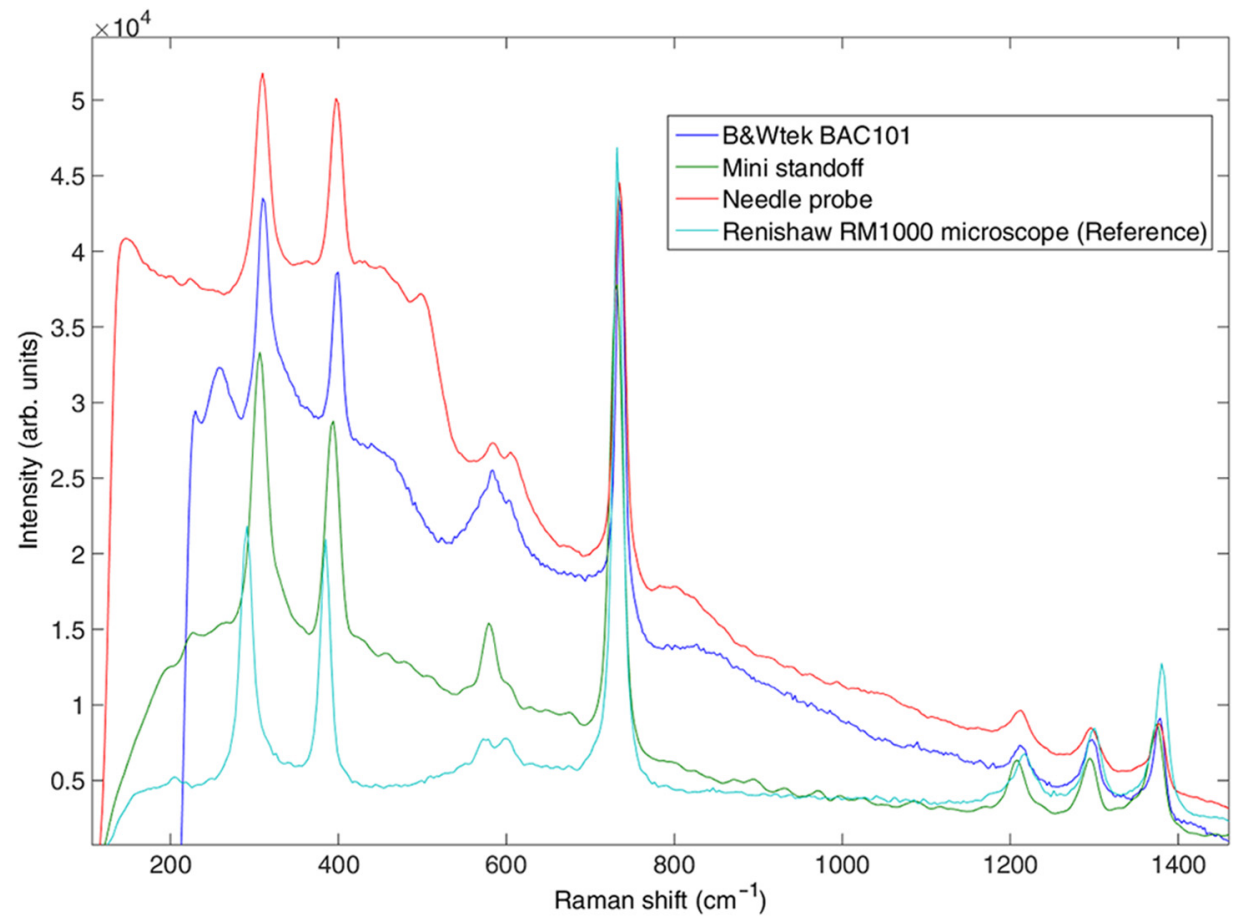

Fig. 4 Raman spectra of the miniature standoff probe and comparable needle probe. Sample laser power was $20 \mathrm{~mW}$, and collection time $30 \mathrm{~s}$. Specta scaled by Raman peak at $385 \mathrm{~cm}^{-1}$ and smoothed with Savitsky-Golay noise filter. The miniature standoff probe has significantly lower background at low wavenumbers than either of the other Raman probes, despite comparable desired Raman signal collection performance. A spectrum from a BAC101 (B\&Wtek, Inc.) handheld Raman probe, and a Raman microscope (RM1000, Renishaw Plc.) are also included for reference.

$G=\iint \mathrm{d} A \mathrm{~d} \Theta$

For a conical beam of light area $S$ bounded by angle $\theta$, this becomes

$G=\pi S \sin ^{2} \theta$.

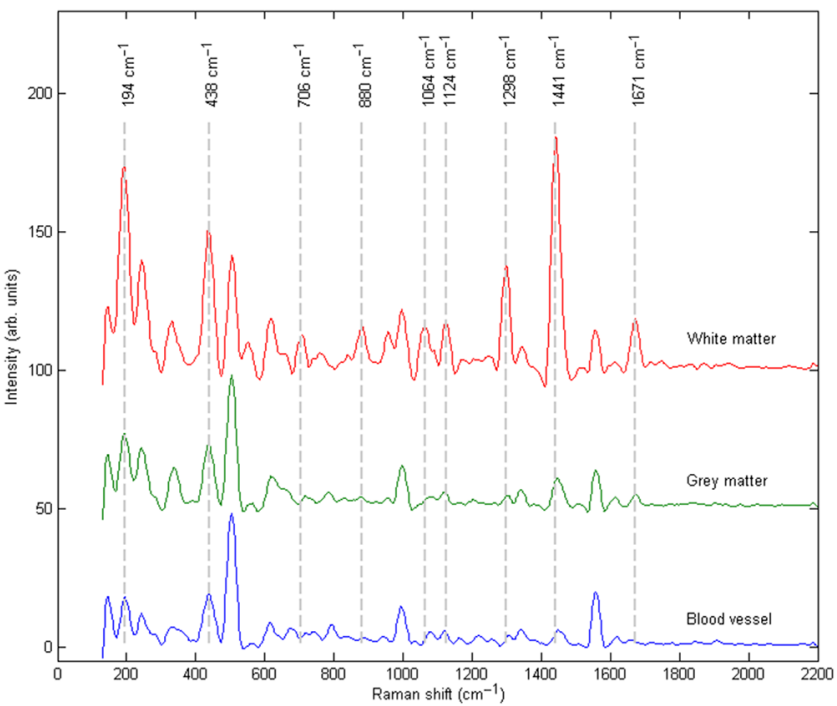

Fig. 5 Raman spectra of porcine brain tissue $(60 \mathrm{~s}, 20 \mathrm{~mW}$, background subtracted). Spectra are offset for illustrative purposes. Clear differences can be seen between the tissue types across the spectral range.
For an optical fiber, the small angle approximation can be used [for small numerical apertures (NA)] to simplify the expression to

$G=\pi A(\mathrm{NA})^{2}$,

where NA is the numerical aperture of the fiber and $A$ is the area of the fiber core.

For the tube in the miniature standoff probe, we must find the amount of light that can pass from one end of the tube to the other. To collect light from an uncoated tube as used in a biopsy needle, there is a negligible component from reflections on the tube walls (waveguiding). In this case, the geometric etendue $(G)$ is found by the "view factor" from a disk of area $A$ representing the entrance of the tube, to another identical disk at the other end. This is expressed as follows: ${ }^{29}$

$G=\pi A F_{1 \rightarrow 2}$

where $F_{1 \rightarrow 2}$ is the view factor between the ends of the needle. This is represented as

$F_{1 \rightarrow 2}=1+\frac{1-\sqrt{4 r^{2}+1}}{2 r^{2}}$

where

$r=\frac{d}{2 l}$

where $d$ is the diameter of the needle and $l$ is the length of the needle. 
Table 1 Example etendues. The miniature standoff probe has a slightly better throughput compared to a $40-\mu \mathrm{m}$ fiber, but lower throughput than larger fibers.

\begin{tabular}{lc} 
Probe & Etendue $\left(\mathrm{m}^{2} \mathrm{sr}\right)$ \\
\hline Mini standoff & $0.161 \times 10^{-9}$ \\
$40-\mu \mathrm{m}$ fiber & $0.157 \times 10^{-9}$ \\
$65-\mu \mathrm{m}$ fiber & $0.416 \times 10^{-9}$ \\
$105-\mu \mathrm{m}$ fiber & $1.09 \times 10^{-9}$ \\
$200-\mu \mathrm{m}$ fiber (needle probe) & $3.95 \times 10^{-9}$ \\
\hline
\end{tabular}

Example numerical etendues for common fiber diameters and the miniature standoff probe are shown in Table 1.

Thus by using the full area of the tip, the total light throughput (etendue) is comparable to a $40-\mu \mathrm{m}$ fiber of NA 0.2 , despite the reduced numerical aperture of 0.005 . The design thus has the advantage of employing no optics at the distal end; the only component at the tip is the steel biopsy needle, which is already approved for medical use. There is an overall light throughput penalty compared to a fiber-based probe in a similar package (equipped with 200- $\mu \mathrm{m}$ fibers), however, the reduced unwanted Raman background results in a similar SNR to a comparable needle probe (Table 2).

The probe operates at an excitation wavelength of $830 \mathrm{~nm}$ to minimize the sample fluorescence.

A dichroic beamsplitter (Semrock, Inc. model LPD01830RU-25) is used to reflect the laser light down the needle, but allow the longer Raman wavelengths to return toward the spectrometer. An additional long wavelength pass filter
(Semrock, Inc. model LPD02-830RU-25) further removes unwanted elastically scattered light from the Raman signal before the collected light is passed into another lens (Thor Labs model F220FC-780), which focuses light into a 100- $\mu \mathrm{m}$ diameter fiber. A laser cleanup filter is (Semrock, Inc. model LL01-830-12.5) also fitted after the laser collimator.

The probe is attached to a Raman explorer spectrometer (Headwall Photonics) and iDus 420BR-DD (Andor Technology) camera controlled with Solis software (Andor Technology).

\section{Testing and Results}

\subsection{Polytetrafluoroethylene (Teflon)}

The probe was tested using a polytetrafluoroethylene (PTFE) reference sample, and compared to a fiber-based needle probe. Fiber background is sample dependent, with reflective samples causing more laser radiation to return into the probe and hence generate significantly more internal Raman background than transmissive (e.g., liquid) samples. PTFE was chosen in this case as it represents a compromise between these two extremes. The needle probe is constructed from low-OH silica fibers of numerical aperture 0.22 - and $200-\mu \mathrm{m}$ diameter. Full details of this probe are described by Day and Stone.$^{30}$ Both probes were connected to the same Raman explorer spectrometer (Headwall Photonics) and iDus 420BR-DD (Andor Technology) camera, via a $1.5-\mathrm{m}$ fiber patch cable. As this patch cable is behind the dichroic filters, it has negligible impact on the internal silica Raman signal generated within the probes. Laser power at the sample was identical between probes $(20 \mathrm{~mW}, 830 \mathrm{~nm}$ at the sample), and acquisition time was set to $30 \mathrm{~s}$ for both probes.

Figure 4 shows a PTFE spectrum taken with the miniature standoff Raman probe, a fiber-based needle probe of the same length $(20 \mathrm{~cm})$, as well as reference spectrum acquired using

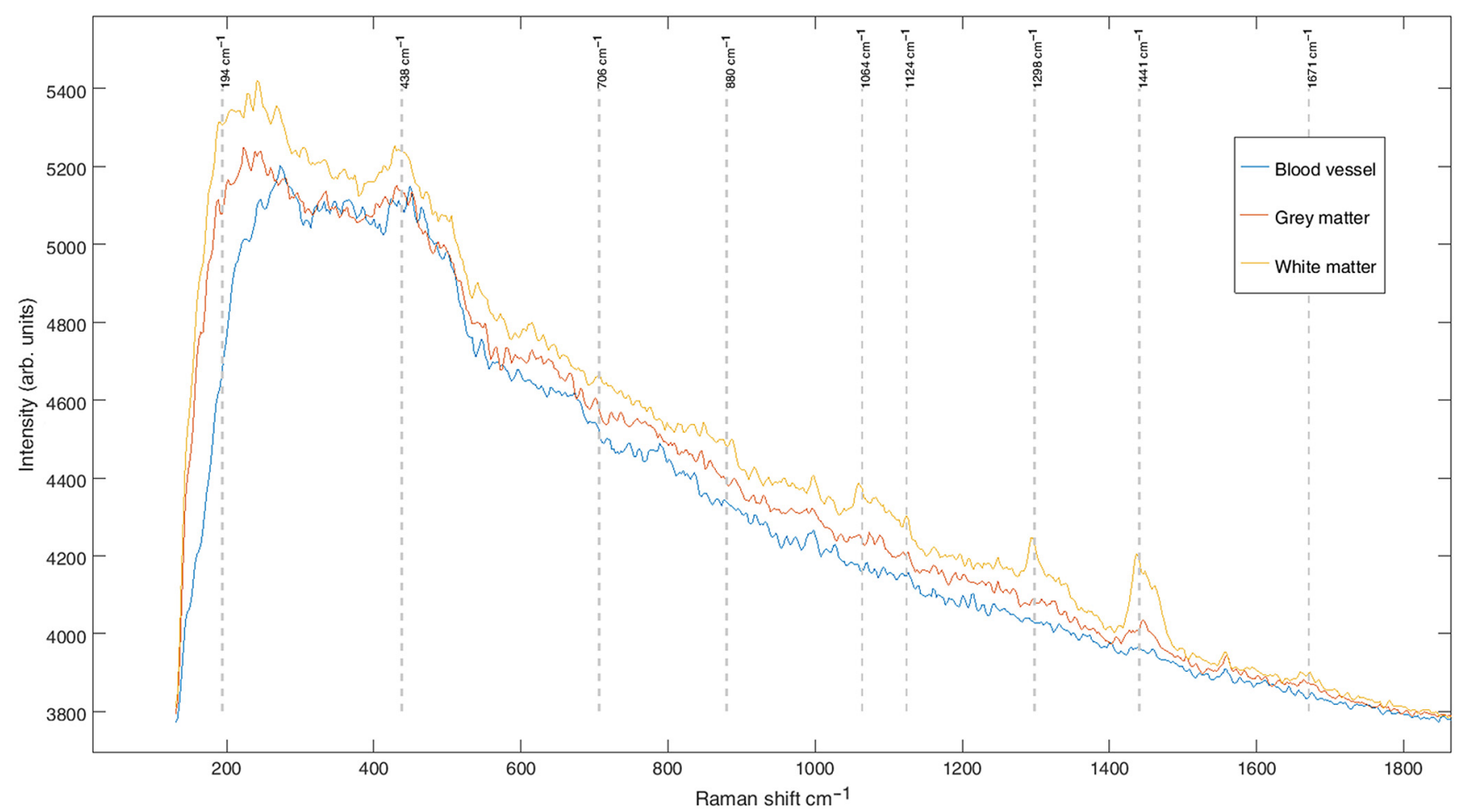

Fig. 6 Raman spectra of porcine brain tissue, as Fig. 5 without background subtraction. 
Table 2 Comparison of quantitative peak to background and peak to noise ratios, at $30-\mathrm{s}$ collection time and $20-\mathrm{mW}$ laser power. The Raman peak (measured at $385 \mathrm{~cm}^{-1}$ ) to background is improved by a factor of around five, and peak to noise is similar between the two probes. Despite a lower throughput, performance is maintained due to greater collection area and a lower internal silica background.

\begin{tabular}{lcc} 
Probe & Peak/background & Peak/noise \\
\hline Mini standoff & 1.75 & 31.9 \\
Needle probe & 0.35 & 31.1 \\
\hline
\end{tabular}

a Renishaw RM1000 Raman microscope $(\sim 5 \mathrm{~mW}, 633 \mathrm{~nm}$, 10 -s integration time, $5 \times$ objective). In addition, a spectrum from a commercially available Raman probe (BAC101, $\mathrm{B} \& W$ tek, Inc.) is included for reference, although this probe is a much larger handheld Raman probe and would be unsuitable for this application. The spectra are scaled by the principal peak at $385 \mathrm{~cm}^{-1}$, which represents the collection performance of desired Raman signal from the sample for each of the probes. The background from the silica of the fiber causes significant additional background at low wavenumbers in the needle probe and the commercial BAC101 probe. In contrast, the miniature standoff Raman probe has a relatively flat background. Peak to background between the needle probe and miniature standoff probe is improved by a factor of five at $385 \mathrm{~cm}^{-1}$. The SNR, calculated from the ratio of the height of the $385 \mathrm{~cm}^{-1}$ peak to the standard deviation of the baseline at $1400 \mathrm{~cm}^{-1}$, is similar between the two probes (Table 2).

\subsection{Use on Porcine Brain}

To demonstrate the effectiveness of the probe on biological samples, it was used to distinguish between different types of healthy brain tissue. Spectra were acquired from porcine brain tissue (obtained from pigs destined for human consumption). One hundred and twenty spectra were acquired of white matter, gray matter, and blood vessels (identified by eye) from a pig brain. A laser excitation of $20 \mathrm{~mW}$ at the sample was used, and an acquisition time of $60 \mathrm{~s}$. Laser power was restricted to $20 \mathrm{~mW}$ due to limitations in available equipement. These spectra are shown with background subtraction applied in Fig. 5, and without subtraction in Fig. 6. The sample fluorescence has been removed from these spectra using a gradient domain approach (outlined in Zhao et al. ${ }^{31}$ ) for clarity. These spectra exhibit the expected Raman bands from brain tissue, previously reported in the literature, ${ }^{23}$ with cholesterol $\left(430,705,1441 \mathrm{~cm}^{-1}\right)$, fatty acid $\left(1130,1298,1441 \mathrm{~cm}^{-1}\right)$, ceramide $\left(1671 \mathrm{~cm}^{-1}\right)$, and sphingomyelin $\left(875 \mathrm{~cm}^{-1}\right)$ Raman peaks all visible. It is also immediately apparent that there are discriminatory differences below $700 \mathrm{~cm}^{-1}$, a region where the silica background of the needle probe is very prevalent, and hence this spectral information would typically be discarded.

\section{Summary and Conclusions}

A miniature Raman probe without fibers in the distal portion of the probe was demonstrated. It employs an approach not previously demonstrated in a Raman probe of this size. By using a collimated beam instead of optical fibers, some throughput is exchanged for a lower background, which can be useful in certain applications. An example of this is brain tissue where there are Raman signatures present at low wavenumber shifts.
While this tradeoff will not be advantageous in all applications, this design offers another option for probe designers in applications where silica background is problematic, or where it is necessary to collect signal from within a needle without optical components at the tip. Additionally, the large spot size at the tip results in a low power density reducing the potential for harm to tissue. Flexibility is not a requirement for use with a stereotactic brain biopsy system, and the miniature standoff design is not flexible as a result; this could be a disadvantage in other applications. The lack of silica fibers greatly increases the medical compatibility of the probe, requiring no additional components to be inserted into the patient. The probe was compared to a silica fiber-based needle probe of similar dimensions, and shown to have similar signal-to-noise performance but much lower background at low wavenumbers, which allows features below $600 \mathrm{~cm}^{-1}$ to be more readily investigated. The suitability of the probe for classification of brain tissue was also demonstrated using porcine tissue. It is anticipated that the probe will be used in the future for optical biopsy of human brain tissue for identification of brain tumor tissue.

\section{Acknowledgments}

The authors would like to thank Severnside Alliance for Translational Research (SATRE) Bio-E initiative and EPSRC for funding this research. Original data for this project is available at http://dx.doi.org/10.5523/bris.zwbmwp6hzes41dhyv2xvz14i1.

\section{References}

1. M. Glas et al., "Residual tumor cells are unique cellular targets in glioblastoma," Ann. Neurol. 68, 264-249 (2010).

2. W. Stummer et al., "Fluorescence-guided surgery with 5-aminolevulinic acid for resection of malignant glioma: a randomised controlled multicentre phase III trial," Lancet Oncol. 7, 392-401 (2006).

3. S. A. Toms et al., "Intraoperative optical spectroscopy identifies infiltrating glioma margins with high sensitivity," Neurosurgery 57, 382-391 (2005).

4. N. Haberland et al., "Neuronavigation in surgery of intracranial and spinal tumors," J. Cancer Res. Clin. Oncol. 126, 529-541 (2000).

5. N. S. Litofsky et al., "Image-guided resection of high-grade glioma: patient selection factors and outcome," Neurosurg. Focus 20, E16 (2006).

6. D. I. Ellis et al., "Illuminating disease and enlightening biomedicine: Raman spectroscopy as a diagnostic tool," Analyst 138, 3871-3884 (2013).

7. A. Nijssen et al., "Towards oncological application of Raman spectroscopy," J. Biophotonics 2, 29-36 (2009).

8. I. Barman et al., "Application of Raman spectroscopy to identify microcalcifications and underlying breast lesions at stereotactic core needle biopsy," Cancer Res. 73(11), 3206-3215 (2013).

9. M. Jermyn et al., "Intraoperative brain cancer detection with Raman spectroscopy in humans," Sci. Transl. Med. 7(274), 274ra19 (2015).

10. K. A. Esmonde-White et al., "Fiber-optic Raman spectroscopy of joint tissues," Analyst 136, 1675-1685 (2011).

11. J. C. C. Day et al., "A miniature confocal Raman probe for endoscopic use," Phys. Med. Biol. 54, 7077-7087 (2009).

12. M. Kirsch et al., "Raman spectroscopic imaging for in vivo detection of cerebral brain metastases," Anal. Bioanal.Chem. 398, 1707-1713 (2010).

13. L. M. Almond et al., "Assessment of a custom-built Raman spectroscopic probe for diagnosis of early oesophageal neoplasia," $J$. Biomed. Opt. 17, 081421 (2012).

14. L. M. Almond et al., "Development of fibre-optic Raman probes for in vivo diagnosis of upper gastrointestinal cancers," Spectrosc. Eur. 23(3), 3-6 (2011).

15. A. T. Harris et al., "Raman spectroscopy in head and neck cancer," Head Neck Oncol 2, 26 (2010). 
16. C. Krafft et al., "Diagnosis and screening of cancer tissues by fiber-optic probe Raman spectroscopy," Biomed. Spectrosc. Imaging 1, 39-55 (2012).

17. J. Mo, W. Zheng, and Z. Huang, "Fiber-optic Raman probe couples ball lens for depth-selected Raman measurements of epithelial tissue," Biomed. Opt. Express 1, 17-30 (2010).

18. A. Mahadevan-Jansen et al., "Rapid communication development of a fiber optic probe to measure NIR Raman spectra of cervical tissue in vivo," Photochem. Photobiol. 68(3), 427-431 (1998).

19. O. Stevens et al., "Developing fibre optic Raman probes for applications in clinical spectroscopy," Chem. Soc. Rev. 45, 1919-1934 (2016).

20. J. Ma and Y. S. Li, "Fiber Raman background study and its application in setting up optical fiber Raman probes," Appl. Opt. 35, 2527-2533 (1996).

21. S. Koljenović et al., "Raman spectroscopic characterization of porcine brain tissue using a single fiber-optic probe," Anal. Chem. 79, 557-564 (2007).

22. L. F. Santos et al., "Fiber-optic probes for in vivo Raman spectroscopy in the high-wavenumber region," Anal. Chem. 77, 6747-6752 (2005).

23. C. Krafft et al., "Near infrared Raman spectra of human brain lipids," Spectrochim. Acta, Part A 61, 1529-1535 (2005).
24. Y. Komachi et al., "Raman probe using a single hollow waveguide," Opt. Lett. 30, 2942-2944 (2005).

25. H. Sato et al., "Raman study of brain functions in live mice and rats: a pilot study," Vib. Spectrosc. 50, 125-130 (2009).

26. S. O. Konorov et al., "Hollow-core photonic crystal fiber-optic probes for Raman spectroscopy," Opt. Lett. 31, 1911-1913 (2006).

27. P. Ghenuche et al., "Kagome hollow-core photonic crystal fiber probe for Raman spectroscopy," Opt. Lett. 37, 4371-4373 (2012).

28. M. Bass, J. M. Enoch, and W. L. Wolfe, Handbook of Optics, McGrawHill, Inc. (2000).

29. A. Bejan and A. D. Kraus, Heat Transfer Handbook, Vol. 1, Wiley (2003).

30. J. C. C. Day and N. Stone, "A subcutaneous Raman needle probe," Appl. Spectrosc. 67, 349-354 (2013).

31. J. Zhao et al., "Automated autofluorescence background subtraction algorithm for biomedical Raman spectroscopy," Appl. Spectrosc. 61, 1225-1232 (2007).

Biographies for the authors are not available. 